\title{
In situ frequency gating and beam splitting of vacuum- and extreme-ultraviolet pulses
}

\author{
Rajendran Rajeev, Johannes Hellwagner, Anne Schumacher, Inga Jordan, Martin Huppert, Andres Tehlar, \\ Bhargava Ram Niraghatam, Denitsa Baykusheva, Nan Lin, Aaron von Conta and Hans Jakob Wörner
}

\begin{abstract}
Monochromatization of high-harmonic sources has opened fascinating perspectives regarding time-resolved photoemission from all phases of matter. Such studies have invariably involved the use of spectral filters or spectrally dispersive optical components that are inherently lossy and technically complex. Here we present a new technique for the spectral selection of near-threshold harmonics and their spatial separation from the driving beams without any optical elements. We discover the existence of a narrow phase-matching gate resulting from the combination of the non-collinear generation geometry in an extended medium, atomic resonances and absorption. Our technique offers a filter contrast of up to $10^{4}$ for the selected harmonics against the adjacent ones and offers multiple temporally synchronized beamlets in a single unified scheme. We demonstrate the selective generation of 133, 80 or $56 \mathrm{~nm}$ femtosecond pulses from a $400-\mathrm{nm}$ driver, which is specific to the target gas. These results open new pathways towards phase-sensitive multi-pulse spectroscopy in the vacuum- and extreme-ultraviolet, and frequencyselective output coupling from enhancement cavities.
\end{abstract}

Light: Science \& Applications (2016) 5, e16170; doi:10.1038/lsa.2016.170; published online 18 November 2016

Keywords: beam splitting; below-threshold harmonics; coherent extreme-ultraviolet pulses; frequency gating; non-collinear generation

\section{INTRODUCTION}

Ultrashort pulses in the vacuum- and extreme-ultraviolet (VUV/XUV) regimes are central to initiating and following ultrafast dynamics of photo-induced reactions ${ }^{1-3}$. The use of low photon energies is particularly attractive because it enables background-free photoemission from electronically excited states. Harmonic emission close to the ionization threshold of the gaseous medium has therefore recently attracted considerable interest ${ }^{4-7}$. An additional motivation for such studies is the rich and incompletely understood mechanism of nearthreshold harmonic (NTH) generation ${ }^{8-12}$. On one hand, the proximity of the corresponding photon energies to the ionization threshold invalidates the traditional treatment of high-harmonic generation based on the strong-field approximation ${ }^{10-12}$. On the other hand, the field control of the characteristic resonance enhancement near bound atomic states has experimentally reaffirmed the nonperturbative nature of the generation mechanism ${ }^{5}$.

The ideal source for ultrafast time-resolved photoemission studies would provide single harmonic orders separated from both the fundamental and the other harmonic orders. The recently introduced non-collinear geometries ${ }^{13-17}$ represent an attractive approach to this goal. Unfortunately, the very large number of possible wave-mixing combinations has prevented their applications so far. As a consequence, dispersive gratings have been required in all of those studies, precluding applications in timeresolved photoemission.
In this article, we demonstrate the new concept of a combined all-optical beam splitter and spectral filter in the VUV/XUV regimes that are built into the generation medium. Our technique combines the unique phase-matching properties of a non-collinear geometry with the effect of a manifold of resonances and absorption in an extended medium. We demonstrate that the emission can be restricted to a single specific harmonic order with a contrast of up to $10^{4}$ allowing for both spatial separation and multi-pulse experiments with multiple synchronized beamlets. Working with a 400-nm driving field, we observe the selective emission of harmonic order three from $\mathrm{Xe}$ and $\mathrm{Kr}$, five from Ar and seven from Ne.

\section{MATERIALS AND METHODS}

The experimental setup is shown in Figure 1. High-harmonic generation from non-collinear wave mixing of linearly polarized driving fields, centered at $400 \mathrm{~nm}$ and focused to intensities of $10^{12}-10^{13} \mathrm{~W} \mathrm{~cm}^{-2}$, was achieved in a semi-infinite gas cell filled with a rare gas. The far-field emission pattern is visualized directly on a micro-channel plate (MCP) without the use of a grating. Multiple independently pumped compartments in conjunction with a combination of horizontal slits enabled differential pumping downstream for safe high-voltage operation of the MCP. The use of 400-nm fields was motivated by the recent experiments on wavelength-scaling of harmonic intensity in the multiphoton regimes ${ }^{18,19}$, which showed that the microscopic harmonic intensity $S_{q}(\lambda)$ scaled with wavelength $\lambda$ 


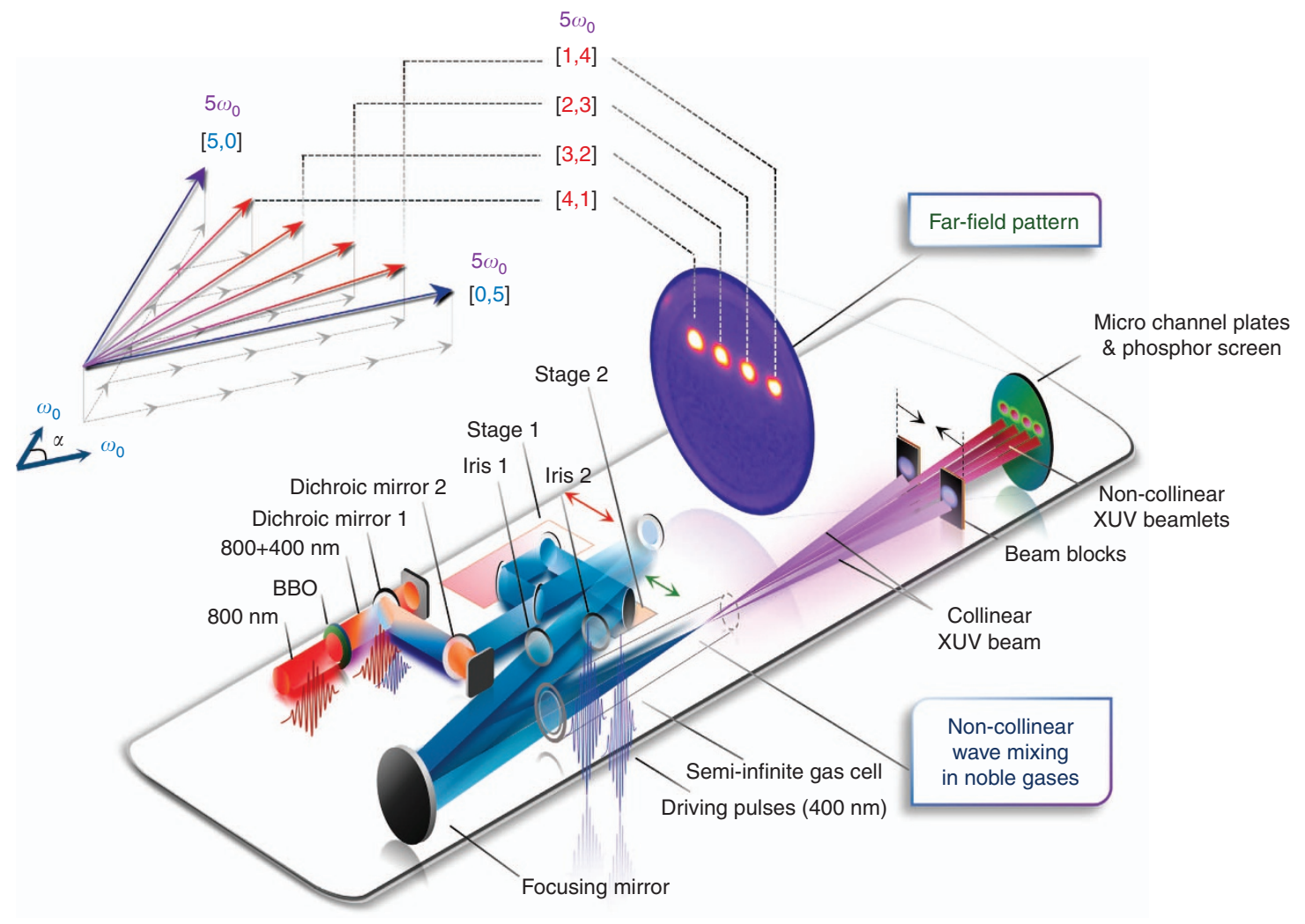

Figure 1 Experimental setup for non-collinear generation of near-threshold harmonics. A 30-fs pulse centered at $800 \mathrm{~nm}$ (red) is frequency doubled in a type-I beta-barium-oxide (BBO) crystal. The 400-nm pulse (blue) is isolated from the infrared beam using a pair of dichroic mirrors and is split into two equally intense beams that are subsequently non-collinearly focused into a semi-infinite gas cell using a $f=50 \mathrm{~cm}$ focusing mirror and spatiotemporally matched using translation stage 1 (red double-headed arrows). Irises 1 and 2 control the diameter and intensity of the driving beams, whereas translation stage 2 (green double-headed arrows) determines the lateral beam separation and thus the crossing angle $\alpha$ in the medium. Non-collinear wave mixing in rare gases generates multiple beamlets in the emission cone that are recorded using a MCP for position-sensitive detection. The beam blocks in front of the detector are used to prevent the intense collinear beams from saturating the MCP. The vector diagram on the top left illustrates the emission directions (arrows in red) based on momentum conservation for $5 \omega_{0}$ beamlets in non-collinear wave mixing of two fields of the same photon energy ( $\left.\omega_{0}\right)$ crossing at an angle $\alpha$. The photon contributions $[m, n]$ from each driving pulse are indicated for the respective beamlets.

as $S_{q}(\lambda) \propto \lambda^{-4.5}$, making a $400-$ nm driver $\sim 23$ times more efficient than $800-\mathrm{nm}$ pulses ${ }^{19}$. In addition, the large inter-harmonic separation of $6.2 \mathrm{eV}$, characteristic of the 400-nm harmonic spectrum, further simplifies their direct spatial separation and spectral characterization without dispersive optical elements. The emission features in the detection plane were resolved by optimizing the diameter of an iris in each of the two driving beams and by introducing beam blocks in the vacuum chamber to prevent the collinear emission from blinding the detector. The order $q$ and photon-number combinations $[m, n]$ of the XUV beamlets within the emission cone were directly identified from their unique lateral positions in the far field (see arrow diagram in Figure 1) as described below. Here and in what follows, $[m, n]$ refers to wave-mixing combinations corresponding to $m$ photons being contributed by one of the driving fields and $n=q-m$ photons by the other.

The intensity of the driving fields were chosen such that NTH dominated the emitted orders. The absence of high harmonics and the presence of only few beamlets thus allowed for their characterization by the unique mapping between lateral position and photon energy $q \omega_{0}$. The emission angle $\beta_{q, m}$ for a given harmonic order $q$ with respect to the bisector of the crossing angle $\alpha$ is given by

$$
\beta_{q, m}=\frac{\alpha}{2}-\tan ^{-1}\left[\frac{m \sin \alpha}{q+m(\cos \alpha-1)}\right]
$$

where $m$ assumes integer values between 0 and $q$. The collinear beam positions on the MCP, measured by removing the beam blocks and lowering the MCP gain, were used to determine the actual angle $\alpha$. For low values of $q$, the angular variation $\Delta \beta$ between beamlets with $[m, n]$ and $[m+1, n-1]$ was large enough to be spatially resolved. The use of driver fields with similar intensities was crucial to implement this map.

The photon flux, given in the caption of Figure 2, was determined as follows. The very low sensitivity of the MCP for the 400-nm fundamental beams makes it an effective transducer for the harmonics. The phosphor screen was simultaneously configured as an anode to measure the total generated charge. The number of photons per pulse $N_{q, \nu}$ contained in one beamlet of harmonic $q$ is related to $N_{q, e}$, the total number of generated electrons as $N_{q, \nu}=N_{q, e} / Q_{q}$, where $Q_{q}$ is the detector quantum efficiency for photons of energy $q \omega_{0}$, provided by the MCP manufacturer. $N_{q, e}$ is related to the integrated fluorescence intensity $I_{q, f}$ of the far-field profile on the phosphor screen as $I_{q, f}=\kappa N_{q, e} G_{V}$ through the MCP gain factor $G_{V}$ (also provided by the manufacturer) at the bias voltage $V$. The constant $\kappa$, which includes the electron-to-photon conversion efficiency of the screen and image acquisition parameters is determined experimentally by selecting one beamlet with the help of physical masks in front of the detector and measuring the total number of electrons that reach the phosphor screen as $N_{q, e} G_{V}=\int J_{q}(t) \mathrm{d} t$, where $J_{q}(t)$ is the measured current. The integrated image intensity is thus related to the current as 
a
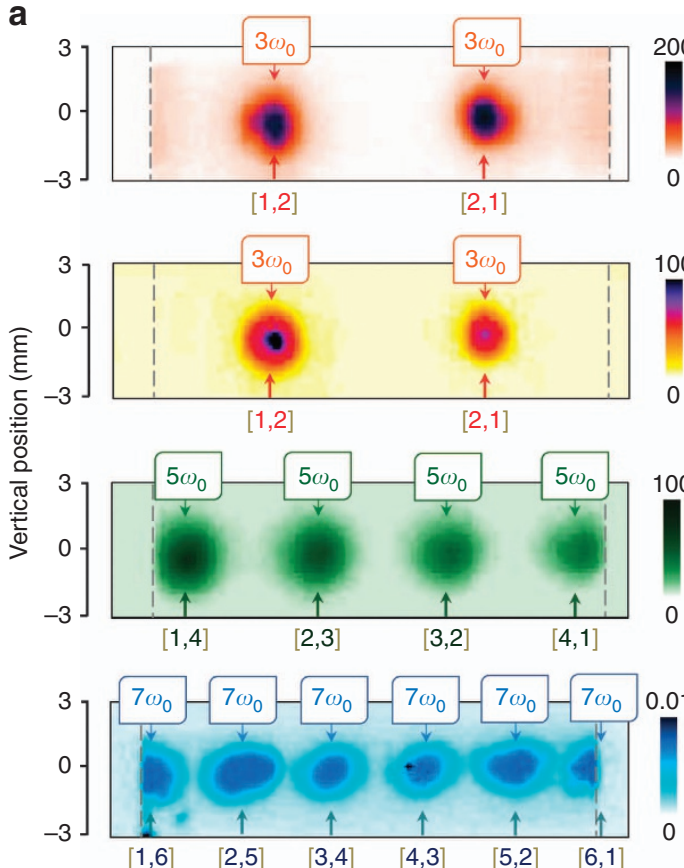

\begin{tabular}{ccccc}
\hline 10 & -5 & 0 & 5 & 10 \\
& Far-field positions & $(\mathrm{mm})$ &
\end{tabular}

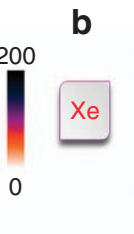

b
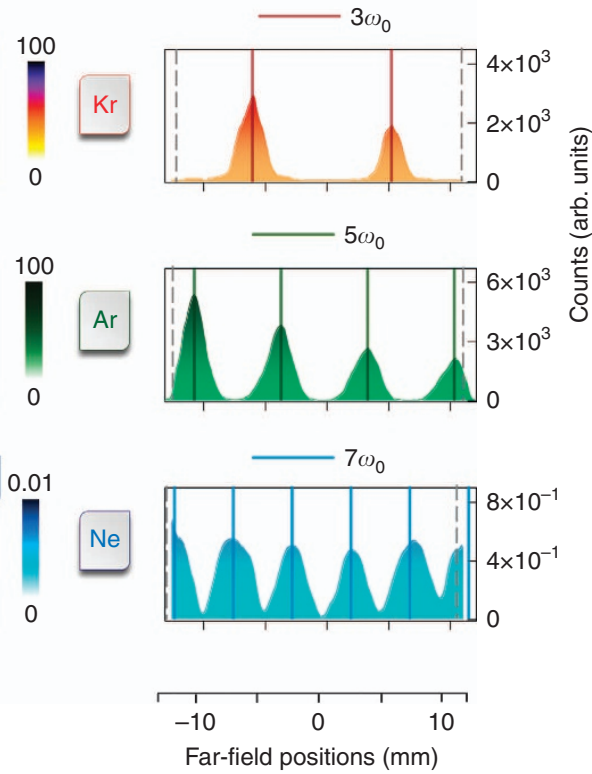

Figure 2 Non-collinear harmonic emission from rare gases. (a) Far-field emission patterns from Xe, Kr, Ar and $\mathrm{Ne}$ recorded under identical experimental conditions. The crossing angle $\alpha$ was chosen to be $\sim 1.8^{\circ}$, and the pressure in the gas cell was maintained at 43 mbar. The relevant harmonics, their expected positions and the corresponding wave-mixing combinations $[m, n]$ are indicated. (b) Vertically integrated line profiles (filled plots) of the far-field patterns indicate gas-specific harmonic emission. The dashed gray lines at the extremes indicate the beam block positions. The beam propagating towards the left was slightly more intense than the other, explaining the weak observed asymmetry. The photon flux of the most intense beamlet was determined to be $7 \times 10^{6}$ photons per pulse for $3 \omega_{0}$ generated in $\mathrm{Xe}, 3 \times 10^{6}$ photons per pulse for $3 \omega_{0}$ generated in $\mathrm{Kr}$ and $1 \times 10^{6}$ photons per pulse for $5 \omega_{0}$ generated in Ar.

$I_{q, f}=\kappa \int J_{q}(t) \mathrm{d} t$ by the constant $\kappa$, enabling us to convert image intensities to photon numbers.

\section{RESULTS AND DISCUSSION}

Figure 2 shows the far-field patterns of harmonic emission from two $400-\mathrm{nm}$ beams using different rare gases as the target. The beams were apertured to a diameter of 3-4 mm using irises, corresponding to pulse energies of $70-90 \mu \mathrm{J}$ and peak intensities of $4-9 \times 10^{12} \mathrm{~W} \mathrm{~cm}^{-2}$. The large Rayleigh range resulting from the focusing geometry $(f / 125$ to $f / 160)$ corresponds to an extended longitudinal overlap with an effective medium length of $l_{\mathrm{m}}=46 \mathrm{~mm}$. The most striking feature in the far-field patterns is the emission of a single harmonic order, characteristic of the target gas. $\mathrm{Xe}$ and $\mathrm{Kr}$ emit only $3 \omega_{0}$, Ar distinctively generates $5 \omega_{0}$ beamlets and $\mathrm{Ne}$ dominantly generates $7 \omega_{0}$ emission. The suppression of above-threshold harmonics $\left(5 \omega_{0}\right.$ and $\left.7 \omega_{0}\right)$ in $\mathrm{Xe}$ and $\mathrm{Kr}$ can be readily understood as a consequence of their strong absorption above the ionization threshold caused by the extended propagation length in the semi-infinite gas cell. However, the absence of below-threshold orders (that is, $3 \omega_{0}$ in Ar, $3 \omega_{0}$ and $5 \omega_{0}$ in $\mathrm{Ne}$ ) with their usual high propensities $S_{q}$ at the singleatom level is unexpected at first sight.

To explain these surprising observations, we now consider in Figure 3 the phase-matching properties of NTH generated in a noncollinear geometry. We illustrate the case of Ar for which the harmonic order $q=5$ falls into the Rydberg manifold converging to the electronic ground state of $\mathrm{Ar}^{+}$. For ease of description, the analysis is restricted to the harmonic orders $3 \omega_{0}, 5 \omega_{0}$ and $7 \omega_{0}$, although the principles are generally valid. The geometric phase mismatch $\Delta k_{\mathrm{g}}$ for the harmonic order $q$ between $k_{\mathrm{h}}$, (the $k$-vector for the harmonic wave) and $k_{\mathrm{p}}$ (the polarization wave resulting from the combined $400-\mathrm{nm}$ driving beams with wave vector $k_{0}$ crossing at an angle $\alpha$ ) is given by Heyl et $a l^{14}$ (also see vector diagram in Figure 3a).

$$
\Delta k_{\mathrm{g}}=k_{0}\left[q-\left(m^{2}+n^{2}+2 m n \cos \alpha\right)^{\frac{1}{2}}\right]
$$

The values of $-\Delta k_{\mathrm{g}}$ for $q=3,5$ and 7 with $m=1$ are shown in Figure 3a (thick lines), along with the atomic phase mismatch $\Delta k_{\mathrm{at}}$ (thin lines) for the experimental conditions $\left(\alpha=1.8^{\circ}\right.$ and gas cell pressure $\sim 43 \mathrm{mbar}$ ) in Ar. The positive Gouy phase mismatch $\Delta k_{\mathrm{G}} \approx 0.05 \mathrm{~cm}^{-1}$ is smaller by more than an order of magnitude. Phase matching of a given harmonic order $q$ is best discussed in terms of a single parameter, the intensity-weighted average of the total phase mismatch $\Delta k_{\mathrm{T}}=\Delta k_{\mathrm{g}}+\Delta k_{\mathrm{at}}$ over the bandwidth $\Delta \omega_{q}$, referred to as $\left\langle\Delta k_{\mathrm{T}}\right\rangle$ and defined as:

$$
\left\langle\Delta k_{\mathrm{T}}\right\rangle=\frac{\int_{\Delta \omega_{q}} \Delta k_{\mathrm{T}}(\omega) I(\omega) \mathrm{d} \omega}{\int_{\Delta \omega_{q}} I(\omega) \mathrm{d} \omega}
$$

Here $I(\omega)$ is the intensity spectrum of the macroscopic response (see below for the evaluation procedure). For the illustrative case of $\operatorname{Ar},\left\langle\Delta k_{\mathrm{T}}\right\rangle$ is shown in Figure $3 \mathrm{~b}$ as a horizontal bar plot. The macroscopic intensity spectrum $I(\omega)$ (Figure $3 \mathrm{~b}$ ) displays 
a

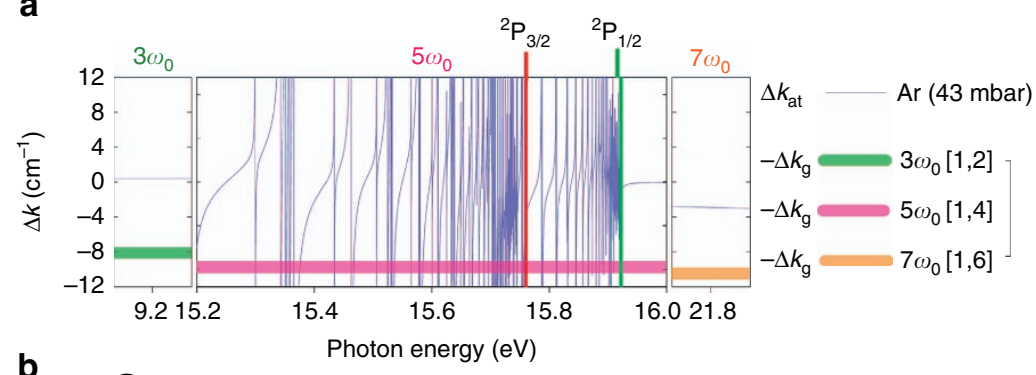

Non-collinear generation geometry $\left(5 \omega_{0}\right)$

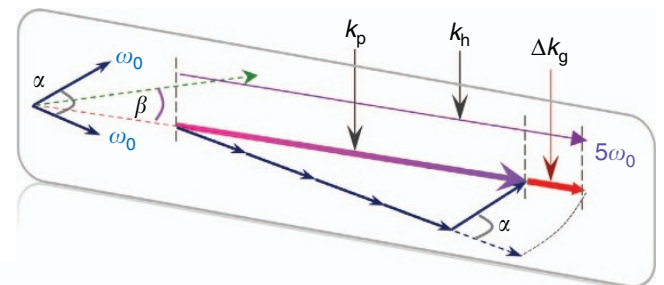

C
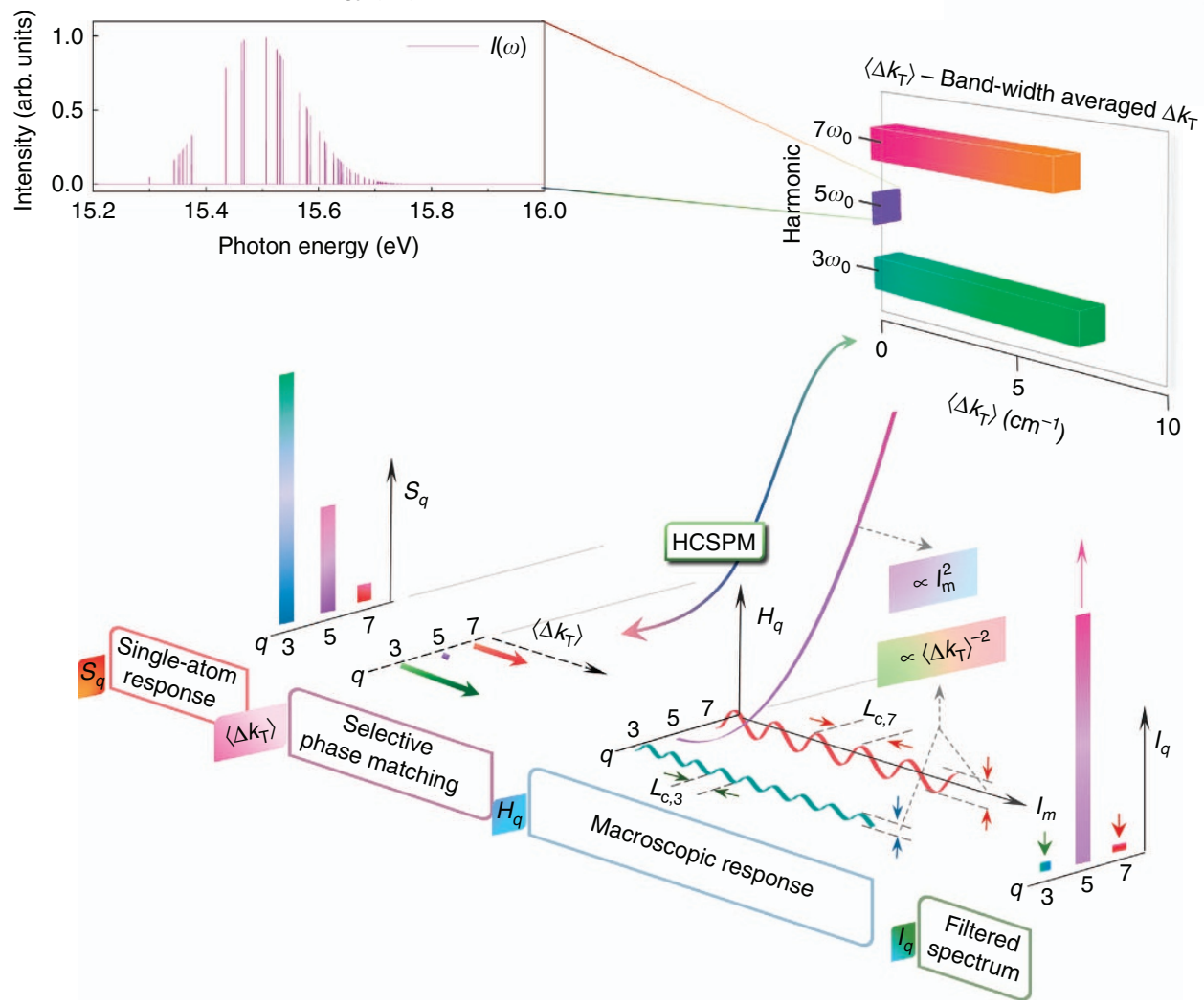

Figure 3 Spectral gating of NTH: the principle in Ar. (a) The geometric and atomic phase mismatches $\Delta k_{\mathrm{g}}$ and $\Delta k_{\mathrm{at}}$ for the case of $400-\mathrm{nm}$ driving beams crossing at an angle $\alpha=1.8^{\circ}$ in Ar at a pressure of $43 \mathrm{mbar}$ are shown over the experimental bandwidth of the harmonics. For clarity, $\Delta k_{\mathrm{g}}$ is considered only for the photon combinations $[1,2],[1,4]$ and $[1,6]$ corresponding to the harmonics $3 \omega_{0}, 5 \omega_{0}$ and $7 \omega_{0}$, respectively. The finite numbers of perfectly phase-matched frequency regions correspond to the points of intersection of $-\Delta k_{\mathrm{g}}$ (thick horizontal lines) with the atomic phase mismatch $\Delta k_{\mathrm{at}}$ (blue line). The vector diagram on the right shows the origin of $\Delta k_{\mathrm{g}}$ in non-collinear geometries towards the generation of $5 \omega_{0}$. (b) Calculated intensity spectrum of the macroscopic response which is dominated by frequency components corresponding to $\Delta k_{T}=0$. The horizontal bar diagram shows the intensity-weighted average $\left\langle\Delta k_{T}\right\rangle$ of the total phase mismatch $\Delta k_{T}=\Delta k_{\mathrm{g}}+\Delta k_{\mathrm{at}}$ over the bandwidth $\Delta \omega_{q}$ of the respective harmonic orders. The near-zero value of $\left\langle\Delta k_{T}\right\rangle$ in the case of harmonic order 5 is attributed to the presence of intensity enhancements at zero crossings of $\Delta k_{T}$ within the Rydberg manifold. (c) Flow diagram illustrating the in situ spectral gating. The single-atom response $S_{q}$ is subjected to High Contrast Selective Phase Matching (HCSPM) in macroscopic propagation, leading to a quadratic growth of $5 \omega_{0}$ over the length of the medium $/ \mathrm{m}$ as reflected by $H_{q}\left(I_{\mathrm{m}}\right)$. The non-phase-matched orders are scaled up in intensity for visibility. The resulting spectrum $I_{q}$ at the end of the medium is monochromatized with high contrast.

pronounced maxima at the phase-matched frequencies within the Rydberg manifold for which $\Delta k_{\mathrm{T}} \approx 0$. The intensity weighting on average therefore leads to $\left\langle\Delta k_{\mathrm{T}}\right\rangle \approx 0$ for the $5 \omega_{0}$ band. We note that the calculated $\left\langle\Delta k_{\mathrm{T}}\right\rangle$ significantly differs from a non-intensityweighted average.

The averaging was performed over the spectral bandwidths measured in a collinear geometry (Supplementary Material; Supplementary Fig. S1). Ordinarily, the phase mismatch $\left\langle\Delta k_{\mathrm{T}}\right\rangle$, varies little from one harmonic order to the next ${ }^{20-22}$. However, in the present case, $\left\langle\Delta k_{\mathrm{T}}\right\rangle$, displays a narrow local minimum at $5 \omega_{0}$. The rapid variation of $\Delta k_{\mathrm{at}}$ with photon energy, resulting from Rydberg resonances, leads to compensation of the large positive values of $\Delta k_{\mathrm{g}}$, and therefore to phase-matched generation at these frequencies. The other orders retain high values of $\left\langle\Delta k_{\mathrm{T}}\right\rangle$, because $\Delta k_{\mathrm{g}}$ cannot be compensated by $\Delta k_{\text {at }}$. We refer to this phenomenon, prevailing in non-collinear generation geometries (see Supplementary Material for a comparison with collinear geometries), as 'High-Contrast Selective Phase Matching' (HCSPM), where phase-matched generation of a given harmonic is simultaneously accompanied by high phase mismatch of the adjacent ones. The observed gas-specific frequency selection of NTH (Figure 2) is a direct consequence of this phenomenon. 
The emitted intensity $I_{q}$ after propagation can be represented as a product of $S_{q}$, the single-atom emission and a macroscopic response function $H_{q}\left(l_{\mathrm{m}}\right)^{23}$ as:

$$
I_{q}=S_{q} H_{q}\left(l_{\mathrm{m}}\right)
$$

In the limit of very low values of the absorption cross-section $\sigma_{q}$, applicable to $\mathrm{NTH}, H_{q}\left(l_{\mathrm{m}}\right)$ has the following limiting functional forms ${ }^{23}$ :

$$
H_{q}\left(l_{\mathrm{m}}\right) \sim \begin{cases}\rho_{\mathrm{m}}^{2} l_{\mathrm{m}}^{2} & \text { for } q=q^{\prime} \\ \rho_{\mathrm{m}}^{2} \frac{2}{\Delta k_{\mathrm{T}}^{2}}\left\{1-\cos \left(\Delta k_{\mathrm{T}} l_{\mathrm{m}}\right)\right\} & \text { for } q=q^{\prime \prime}\end{cases}
$$

Here $q^{\prime}$ corresponds to a phase-matched harmonic order, $q^{\prime \prime}$ is a nonphase-matched order and $\rho_{\mathrm{m}}$ is the medium density. Whereas the intensity of the phase-matched order grows quadratically within the medium, the intensity of the adjacent harmonics oscillates between a maximum value of $H_{q^{\prime \prime}, \max }=\left(4 / \pi^{2}\right) \rho_{\mathrm{m}}^{2} L_{c, q^{\prime \prime}}^{2}$ and zero (Figure $3 \mathrm{c}$ ). Here $L_{\mathrm{c}, q^{\prime \prime}}=\pi / \Delta k_{\mathrm{T}}$ is the coherence length of the non-phase-matched orders $q^{\prime \prime}$. Note that $H_{q^{\prime \prime}, \max }$ is inversely proportional to $\Delta k_{\mathrm{T}}^{2}$, providing an independent and sensitive handle for determining the maximum intensity growth within the medium for $q^{\prime \prime}$. The large values of $\left\langle\Delta k_{\mathrm{T}}\right\rangle$ for the non-phase-matched orders suppress their intensities and hence allow for frequency gating of harmonic emission to a single order at the end of the medium.

Figure $3 c$ summarizes these concepts for the case of argon. The single-atom intensity distribution $S_{q}$ is subjected to HCSPM and the macroscopic response function $H_{q}\left(l_{\mathrm{m}}\right)$ restricts the beamlets in the emission cone to solely the phase-matched order $5 \omega_{0}$ in the final spectrum $I_{q}$. Applying the same principle to neon explains the selective generation of $7 \omega_{0}$ and the suppression of $3 \omega_{0}$ and $5 \omega_{0}$, leading to the emission of six synchronized beams with a photon energy of $21.7 \mathrm{eV}$, as observed. This technique relies on (a) enhancing $\left\langle\Delta k_{\mathrm{T}}\right\rangle$ for the nonNTH orders and (b) large propagation lengths to translate HCSPM to a high degree of spectral contrast in $I_{q}$. While in the non-collinear geometry, the use of $400-\mathrm{nm}$ light fields with enhanced $k_{0}$ (Equation (2)) and the overlap of $q \omega_{0}$ with the Rydberg manifold fulfills condition (a), a large wave-mixing length $l_{\mathrm{m}}$ (possible in semi-infinite gas cell) with small crossing angle $\alpha$ was crucial towards condition (b) and observing this mechanism in our experiments. A comparison of spectral gating in collinear and non-collinear geometries is shown in the Supplementary Material (Supplementary Fig. S2).

To validate these principles further, we have performed calculations to evaluate the spectral composition of the non-collinear beamlets after propagation and show the results in Figure 4. These calculations take into account all phase-matching characteristics as well as absorption by the medium in calculating the harmonic intensities $I_{q}(\omega)$. Arriving at $I_{q}(\omega)$ involved the experimental determination of $S_{q}(\omega)$ and the calculation of $H_{q}(\omega)$.

The atomic response $S_{q}$ was directly measured in complementary experiments using a collinear geometry (Supplementary Material; Supplementary Fig. S1) and was represented by:

$$
S_{q}(\omega)=A_{q} \exp \left[-\left(\frac{\omega-\omega_{q}}{q \Delta \omega_{0}}\right)^{2 q}\right]
$$

Here $\Delta \omega_{0}$ is the full-width-at-half-maximum bandwidth of the spectrum of the fundamental driving pulse. The pre-factors $A_{q}$ were obtained from an experimental determination of the integrated singleatom response $S_{q}=\int S_{q}(\omega) \mathrm{d} \omega$ (Supplementary Material). The atomic contribution towards the macroscopic response function $H_{q}(\omega)=$ $H_{q}\left(l_{\mathrm{m}} ; \rho_{\mathrm{m}} ; \sigma_{q}(\omega) ; \Delta k_{\mathrm{T}}(\omega)\right)$ depends on the atomic phase mismatch
$\Delta k_{\mathrm{at}}(\omega)=k(\omega)-q k\left(\omega_{0}\right)$ and absorption $\sigma(\omega)$, where $k(\omega)$ and $\sigma(\omega)$ are the dispersion relationship and the frequency-dependent absorption in the medium, respectively. $k(\omega)$ and $\sigma(\omega)$ are related to the real and imaginary parts, respectively, of the complex refractive index $n(\omega)=n_{\mathrm{R}}(\omega)+\mathrm{i} n_{\mathrm{I}}(\omega)$ as follows:

$$
k(\omega)=\frac{2 \pi n_{\mathrm{R}}(\omega)}{\lambda(\omega)}
$$

and

$$
\sigma(\omega)=\frac{4 \pi n_{\mathrm{I}}(\omega)}{\rho_{\mathrm{m}} \lambda(\omega)}
$$

Here and in what follows below, $\rho_{\mathrm{m}}$ is the atomic density and $\omega_{\mathrm{th}}$ is the energy corresponding to the ionization threshold in atomic units. Both the real and imaginary parts of the refractive index have contributions from the continuum and from atomic resonances located at photon energies $\omega_{j}$. The resonance contributions are calculated within the framework of the Lorentz oscillator model for polarization using the following coupled equations:

$$
n_{\mathrm{R}, \text { line }}^{2}(\omega)-n_{\mathrm{I}, \text { line }}^{2}(\omega)=1+\frac{\rho_{\mathrm{m}} e^{2}}{m \epsilon_{0}} \sum_{j} \frac{f_{j}\left(\omega_{j}^{2}-\omega^{2}\right)}{\left(\omega_{j}^{2}-\omega^{2}\right)^{2}+\Gamma_{j}^{2} \omega^{2}}
$$

and

$$
2 n_{\mathrm{R}, \text { line }}(\omega) n_{\mathrm{I}, \text { line }}(\omega)=\frac{\rho_{\mathrm{m}} e^{2}}{m \epsilon_{0}} \sum_{j} \frac{f_{j} \Gamma_{j} \omega}{\left(\omega_{j}^{2}-\omega^{2}\right)^{2}+\Gamma_{j}^{2} \omega^{2}}
$$

The continuum contributions are given by

$$
\begin{gathered}
n_{\mathrm{R}, \text { cont }}(\omega)=1+\frac{N}{\pi} \int_{\omega_{\text {th }}}^{\infty} \frac{\sigma_{\text {ioni }}\left(\omega_{i}\right) \mathrm{d} \omega_{i}}{\omega_{i}^{2}-\omega^{2}} \\
n_{\mathrm{I}, \text { cont }}(\omega)=\sigma_{\text {ioni }}(\omega) \rho_{m} \lambda(\omega) / 4 \pi
\end{gathered}
$$

Here $f_{j}$ is the oscillator strength for the $j^{\text {th }}$ dipole-allowed transition of photon energy $\omega_{j}$. Further, $\Gamma_{j}=1 / \tau_{j}$ is the natural linewidth of the transition, $e$ and $m$ are the charge and mass of electron, $\epsilon_{0}$ is the permittivity of vacuum, $\sigma_{\text {ioni }}(\omega)$ is the single-photon ionization crosssection at photon energy $\omega$.

In the calculations, we used a generation length equal to the longitudinal beam overlap of $46 \mathrm{~mm}$, an additional effective length for absorption of $10 \mathrm{~mm}$, a crossing angle $\alpha=1.8^{\circ}$ and a pressure of 43 mbar. The spectral evaluation of intensities $I(\omega)$ near the threshold was performed over a grid with a step size of $25 \mu \mathrm{eV}$ small enough to resolve resonances. The predicted intensities $I_{q}(\omega)$ are shown in Figure $4 \mathrm{~b}$.

Overall, the calculated intensities indeed predict the generation of a single dominant order, specific to the chosen medium. The enhancement of $5 \omega_{0}$ in $\mathrm{Ar}$ and $7 \omega_{0}$ in Ne is caused by their overlap with Rydberg manifolds converging to their lowest ionization thresholds and leads to the formation of resonance-enhanced structures observed in collinear 400-nm harmonic generation (Supplementary Material; Supplementary Fig. S1). The proximity of $3 \omega_{0}$ to the position of the 6-s resonance of $\mathrm{Xe}(9.56973 \mathrm{eV})$ causes a rapid variation of the phase mismatch $\Delta k_{\mathrm{T}}$ across the bandwidth of the harmonic, high enough to modulate the spectrum. In $\mathrm{Kr}$, the harmonic $3 \omega_{0}$ is further away from the closest 5-s resonance $(10.0324 \mathrm{eV})$ and hence has a lower variation in $\Delta k_{\mathrm{T}}$ that leads to a weaker modulation of the spectrum. In both cases, however, the strong absorption just above the ionization threshold plays an assisting role by additionally suppressing the intensities of above-threshold orders. 


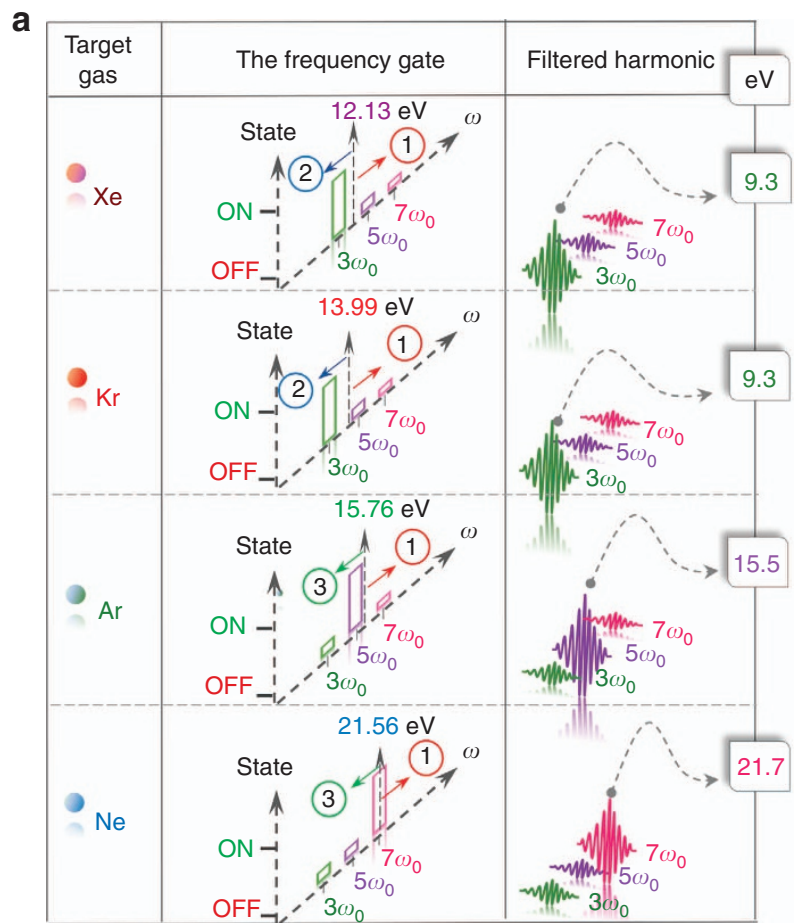

(1) Absorption dominated (2) Non-phase matched generation (3) HCSPM b

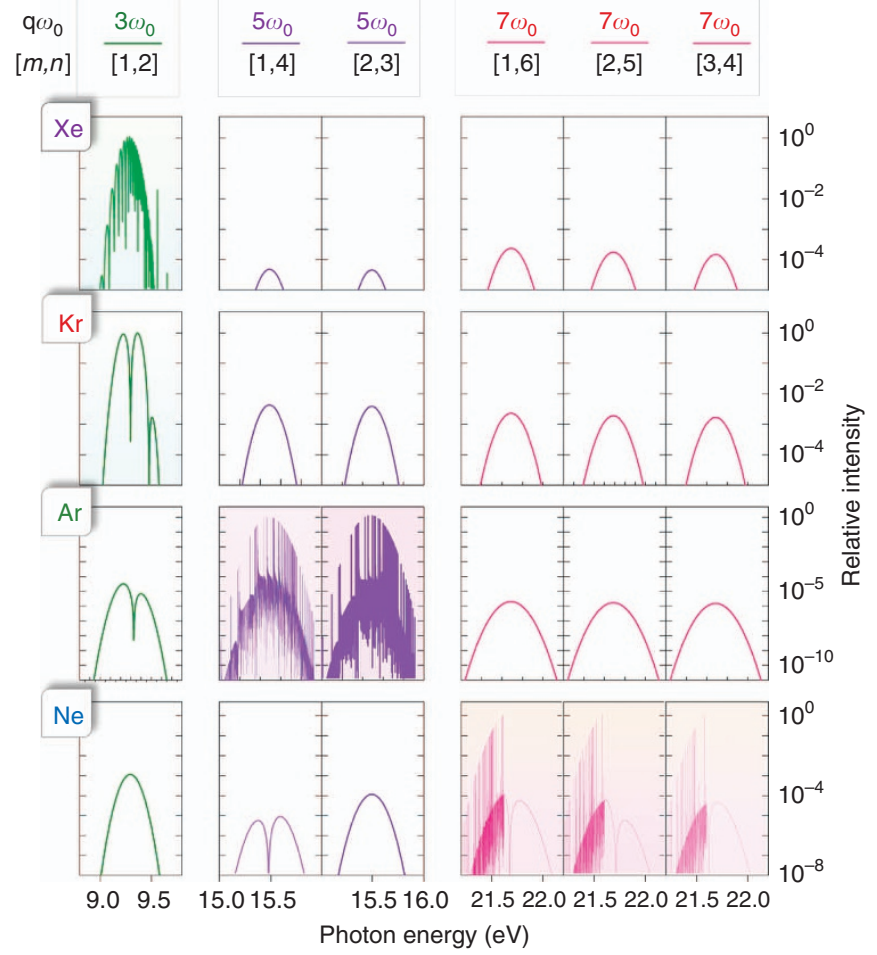

Figure 4 NTH frequency gating and tunability in rare gases. (a) The spectral gating (rectangles in column 2) in non-collinear emission can be tuned by changing the generation gas leading to selection of $9.3 \mathrm{eV}$ (in $\mathrm{Xe}$ and $\mathrm{Kr}$ ), $15.5 \mathrm{eV}$ (in $\mathrm{Ar}$ ) and $21.7 \mathrm{eV}$ (in $\mathrm{Ne}$ ) photons. A rectangle with large height ('on' state) represents the presence and shorter rectangles ('off' state) the absence of a specified harmonic order in the non-collinear emission. The vertical arrow for each target gas corresponds to the respective ionization limit. Further, the circled numbers highlight the dominant mechanisms in each spectral zone (diagonal blue, red and green arrows) for the respective gas. Column 3 pictorially shows the spectral content of the harmonic emission. (b) Predicted spectral intensities $I_{q}(\omega)$ for rare gases for three different orders $q$ and their respective wave-mixing combinations $[m, n]$.

Finally, we studied the duration of the generated VUV/XUV pulses. We measured the duration of $3 \omega_{0}$ pulses generated in $\mathrm{Xe}$ and $5 \omega_{0}$ pulses generated in $\mathrm{Ar}$ in a collinear geometry to be $\sim 78 \mathrm{fs}$ (Supplementary Material; Supplementary Fig. S4). Measurements performed in a non-collinear geometry for $5 \omega_{0}$ pulses generated in Ar lead to a consistent result (Supplementary Material; Supplementary Fig. S3). The spectrally-resolved far-field profile from Ar is shown in the Supplementary Material (Supplementary Fig. S5). These results show that the presence of resonances does not prevent the emission of ultrashort VUV/XUV laser pulses that will therefore find applications in time-resolved photoemission experiments.

\section{CONCLUSIONS}

In summary, we devised a new scheme for all-optical frequency gating and simultaneous beam splitting of VUV/XUV radiation inside the generation medium. Our scheme exploits the phasematched generation facilitated by resonances close to the ionization limit and the concomitant phase mismatch of adjacent harmonics in a non-collinear geometry. The scheme of HCSPM introduced in this article can transform the monotonically decreasing single-atom response $S_{q}$ to a spectrally filtered function $I_{q}$ as a consequence of the non-collinear macroscopic response function $H_{q}$. We demonstrated both experimentally and theoretically that the effective realization of HCSPM requires a non-collinear geometry. The high contrast of the filter relies on the long medium length $l_{\mathrm{m}}$ made possible in a semi-infinite gas cell. The scheme of HCSPM reported here is generally applicable and can be used to spectrally filter a range of photon energies within the Rydberg manifolds. This scheme could become an alternative to the conventional dispersion-based grating monochromators ${ }^{24}$ or multilayer mirrors ${ }^{25}$ due to its all-optical nature and simple layout. The non-collinear filter has the added advantage of naturally separating the harmonic radiation from the fundamental and the availability of multiple synchronized beamlets for multi-pulse pump-probe schemes ${ }^{26,27}$. Consequently, this scheme may enable new approaches to frequency-selective outcoupling from femtosecond enhancement cavities $^{28-30}$, paving the way to $\mathrm{MHz}$ repetition-rate spectrally pure XUV sources with possible applications in ultrafast time-resolved spectroscopy, XUV holography and microscopy.

\section{CONFLICT OF INTEREST}

The authors declare no conflict of interest.

\section{ACKNOWLEDGEMENTS}

We gratefully acknowledge support from a starting grant (project No. 307270ATTOSCOPE) of the European Research Council and the Swiss National Science Foundation via the National Centre of Competence in Research Molecular Ultrafast Science and Technology.

1 Nugent-Glandorf L, Scheer M, Samuels DA, Mulhisen AM, Grant ER et al. Ultrafast time-resolved soft X-ray photoelectron spectroscopy of dissociating $\mathrm{Br}_{2}$. Phys Rev Lett 2001; 87: 193002. 
2 Siefermann KR, Liu YX, Lugovoy E, Link O, Faubel M et al. Binding energies, lifetimes and implications of bulk and interface solvated electrons in water. Nat Chem 2010; 2 274-279.

3 Dachraoui H, Michelswirth M, Siffalovic P, Bartz P, Schäfer C et al. Photoinduced reconfiguration cycle in a molecular adsorbate layer studied by femtosecond inner-shel photoelectron spectroscopy. Phys Rev Lett 2011; 106: 107401.

4 Benko C, Allison TK, Cingöz A, Hua LQ, Labaye F et al. Extreme ultraviolet radiation with coherence time greater than 1 s. Nat Photon 2014; 8: 530-536.

5 Chini M, Wang XW, Cheng Y, Wang $\mathrm{H}$, Wu Y et al. Coherent phase-matched VUV generation by field-controlled bound states. Nat Photon 2014; 8: 437-441.

6 Brizuela F, Heyl CM, Rudawski P, Kroon D, Rading L et al. Efficient high-order harmonic generation boosted by below-threshold harmonics. Sci Rep 2013; 3: 1410.

7 Hammond TJ, Mills AK, Jones DJ. Near-threshold harmonics from a femtosecond enhancement cavity-based EUV source: effects of multiple quantum pathways on spatial profile and yield. Opt Express 2011; 19: 24871-24883.

8 Yost DC, Schibli TR, Ye J, Tate JL, Hostetter J et al. Vacuum-ultraviolet frequency combs from below-threshold harmonics. Nat Phys 2009; 5: 815-820.

9 Power EP, March AM, Catoire F, Sistrunk E, Krushelnick K et al. XFROG phase measurement of threshold harmonics in a Keldysh-scaled system. Nat Photon 2010; 4: 352-356.

10 Hostetter JA, Tate JL, Schafer KJ, Gaarde MB. Semiclassical approaches to belowthreshold harmonics. Phys Rev A 2010; 82: 023401.

11 Soifer H, Botheron P, Shafir D, Diner A, Raz O et al. Near-threshold high-order harmonic spectroscopy with aligned molecules. Phys Rev Lett 2010; 105: 143904.

12 Xiong WH, Geng JW, Tang JY, Peng LY, Gong Q. Mechanisms of below-threshold harmonic generation in atoms. Phys Rev Lett 2014; 112: 233001.

13 Bertrand JB, Wörner HJ, Bandulet HC, Bisson É, Spanner M et al. Ultrahigh-order wave mixing in non-collinear high harmonic generation. Phys Rev Lett 2011; 106: 023001.

14 Heyl CM, Rudawski P, Brizuela F, Bengtsson SN, Mauritsson J et al. Macroscopic effects in non-collinear high-order harmonic generation. Phys Rev Lett 2014; 112 143902.

15 Heyl CM, Bengtsson S, Carlström S, Mauritsson J, Arnold C et al. Non-collinear optical gating. New J Phys 2014; 16: 052001.

16 Louisy M, Arnold CL, Miranda M, Larsen EW, Bengtsson SN et al. Gating attosecond pulses in a non-collinear geometry. Optica 2015; 2: 563-566.

17 Hickstein DD, Dollar FJ, Grychtol P, Ellis JL, Knut R et al. Non-collinear generation of angularly isolated circularly polarized high harmonics. Nat Photon 2015; 9: 743-750.

18 Wang H, Xu YM, Ulonska S, Robinson JS, Ranitovic P et al. Bright high-repetition-rate source of narrowband extreme-ultraviolet harmonics beyond $22 \mathrm{eV}$. Nat Commun 2015; 6: 7459.
19 Lai CJ, Cirmi G, Hong KH, Moses J, Huang SW et al. Wavelength scaling of high harmonic generation close to the multiphoton ionization regime. Phys Rev Lett 2013 111: 073901.

20 Rundquist A, Durfee CG, Chang ZH, Herne C, Backus S et al. Phase-matched generation of coherent soft X-rays. Science 1998; 280: 1412-1415.

21 Paul A, Bartels RA, Tobey R, Green H, Weiman S et al. Quasi-phase-matched generation of coherent extreme-ultraviolet light. Nature 2003; 421: 51-54.

22 Popmintchev T, Chen MC, Arpin P, Murnane MM, Kapteyn HC. The attosecond nonlinear optics of bright coherent X-ray generation. Nat Photon 2010; 4: 822-832.

23 Constant E, Garzella D, Breger P, Mével E, Dorrer C et al. Optimizing high harmonic generation in absorbing gases: model and experiment. Phys Rev Lett 1999; 82 1668-1671.

24 Haelbich RP, Kunz C. Multilayer interference mirrors for the XUV range around $100 \mathrm{eV}$ photon energy. Opt Commun 1976; 17: 287-292.

25 Frassetto $F$, Cacho C, Froud CA, Turcu ICE, Villoresi $P$ et al. Single-grating monochromator for extreme-ultraviolet ultrashort pulses. Opt Express 2013; 19 : 19169-19181.

26 Bencivenga F, Cucini R, Capotondi F, Battistoni A, Mincigrucci R et al. Four-wave mixing experiments with extreme ultraviolet transient gratings. Nature 2015; 520: 205-208.

27 Kowalewski M, Bennett K, Dorfman KE, Mukamel S. Catching conical intersections in the act: monitoring transient electronic coherences by attosecond stimulated X-ray raman signals. Phys Rev Lett 2015; 115: 193003.

28 Gohle C, Udem T, Herrmann M, Rauschenberger J, Holzwarth R et al. A frequency comb in the extreme ultraviolet. Nature 2005; 436: 234-237.

29 Krischek R, Wieczorek W, Ozawa A, Kiesel N, Michelberger P et al. Ultraviolet enhancement cavity for ultrafast nonlinear optics and high-rate multiphoton entanglement experiments. Nat Photonics 2010; 4: 170-173.

30 Yost DC, Schibli TR, Ye J. Efficient output coupling of intra-cavity high-harmonic generation. Opt Lett 2008; 33: 1099-1101.

\section{(c) (i)}

This work is licensed under a Creative Commons Attribution 4.0 International License. The images or other third party material in this article are included in the article's Creative Commons license, unless indicated otherwise in the credit line; if the material is not included under the Creative Commons license, users will need to obtain permission from the license holder to reproduce the material. To view a copy of this license, visit http://creativecommons.org/licenses/by/4.0/

(C) The Author(s) 2016

Supplementary Information for this article can be found on the Light: Science \& Applications' website (http://www.nature.com/lsa). 